\title{
The Exponential Map Near Conjugate Points In 2D Hydrodynamics
}

\author{
Gerard Misiołek $^{1,2}$
}

Received: 28 November 2014 / Accepted: 15 June 2015 / Published online: 1 July 2015

(C) Institute for Mathematical Sciences (IMS), Stony Brook University, NY 2015

\begin{abstract}
We prove that the weak-Riemannian exponential map of the $L^{2}$ metric on the group of volume-preserving diffeomorphisms of a compact two-dimensional manifold is not injective in any neighbourhood of its conjugate vectors. This can be viewed as a hydrodynamical analogue of the classical result of Morse and Littauer.
\end{abstract}

Keywords Exponential map - Diffeomorphisms · Fredholm map · Conjugate points

Mathematics Subject Classification Primary 58B25 - 46T05;

Secondary $37 \mathrm{~K} 65 \cdot 35 \mathrm{Q} 35$

\section{Introduction}

Let $M$ be an $n$-dimensional compact Riemannian manifold. Suppose that $M$ is filled with an incompressible inviscid fluid whose particles cannot fuse or split. The Lagrangian approach to hydrodynamics aims at describing trajectories of these particles as they trace out a geodesic of a right-invariant metric defined by fluid's kinetic energy in the group of Sobolev class diffeomorphisms $\mathscr{D}_{\mu}^{s}=\mathscr{D}_{\mu}^{s}(M)$ preserving the volume form $\mu$ (the volumorphism group). The metric is given at the identity diffeomorphism $e$ by the $L^{2}$ inner product

$$
\langle v, w\rangle_{L^{2}}=\int_{M}\langle v(x), w(x)\rangle d \mu
$$

$\bowtie$ Gerard Misiołek gmisiole@nd.edu

1 Department of Mathematics, University of Colorado, Boulder, CO 80309-0395, USA

2 Department of Mathematics, University of Notre Dame, Notre Dame, IN 46556, USA 
where $v, w \in T_{e} \mathscr{D}_{\mu}^{s}$ are divergence free Sobolev $H^{s}$ vector fields on $M$. This metric is only weak-Riemannian ${ }^{1}$ in that the tangent spaces to $\mathscr{D}_{\mu}^{s}$ with the induced inner products are (incomplete) pre-Hilbert spaces. Its geodesics are the critical points of the associated $L^{2}$ length functional

$$
L(\eta)=\int_{0}^{T}\|\dot{\eta}(t)\|_{L^{2}} d t
$$

and satisfy the equations

$$
\frac{d^{2} \eta}{d t^{2}}=-\operatorname{grad} p \circ \eta
$$

which rewritten on the Lie algebra of divergence-free vector fields become the Euler equations of hydrodynamics

$$
\begin{aligned}
\frac{\partial v}{\partial t}+v \cdot \nabla v & =-\operatorname{grad} p \\
\operatorname{div} v & =0
\end{aligned}
$$

where $p$ is the pressure function of the fluid, $v=\dot{\eta} \circ \eta^{-1}$ and $v \cdot \nabla$ denotes the covariant derivative on $M$ in the direction of $v$.

As in finite-dimensional differential geometry the weak-Riemannian metric on $\mathscr{D}_{\mu}^{s}$ defined by (1.1) has an exponential map however its global properties are not yet fully understood. Recall that two points $p$ and $q=\exp _{p} v$ along a geodesic are conjugate if the derivative $d \exp _{p}(v)$ fails to be an isomorphism. In infinite dimensions this may happen if either the kernel is non-empty or the range is not the whole target space. Accordingly, one has to distinguish mono-conjugate points and epi-conjugate points, respectively. Roughly speaking, the former are related to minimizing properties of geodesics while the latter to covering properties of the exponential map. What further complicates matters is the fact that conjugate points (of either type) can accumulate along finite geodesic segments or that their multiplicities can be of infinite order, see e.g. Grossman (1965).

However, even in finite dimensions singularities of Riemannian exponential maps are of a rather special kind. Morse and Littauer (1932) proved that a singularity of $d \exp _{p}(v)$ necessarily implies non-injectivity of the exponential map near the vector $v$ for any analytic Riemannian or Finslerian manifold. Savage (1943) reproved this result in the smooth case and Warner (1965) extended it to a more general class of maps. More recently, Biliotti et al. (2006) revisited it in the context of strong-Riemannian Hilbert manifolds.

It turns out that there is a hydrodynamical analogue of the Morse-Littauer result. Namely, our main goal is to prove the following

Theorem Let $M$ be a smooth closed Riemannian manifold of dimension 2 and assume $s>2$. Consider a geodesic $\eta(t)$ in $\mathscr{D}_{\mu}^{s}$ of the $L^{2}$ metric (1.1) starting from the identity

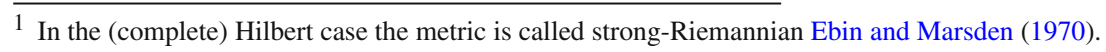


$e$ with velocity $v_{0}$ and let $\eta\left(t_{c}\right)$ be the first conjugate point to $e$. Then the weakRiemannian $L^{2}$ exponential map is not injective at $t_{c} v_{0}$.

In particular, we have the following simple hydrodynamical interpretation: every neighbourhood of $t_{c} v_{0}$ in $T_{e} \mathscr{D}_{\mu}^{s}$ contains a vector whose image under the exponential map corresponds to a configuration of a $2 \mathrm{D}$ fluid which can be reached from the initial state by two distinct fluid flows in the same amount of time.

An important fact used in the proof below is that the weak-Riemannian exponential map in 2D hydrodynamics is a Fredholm map of index zero. Combined with a hydrodynamical Morse Index theorem and a Schauder type invariance of domain result this fact allows us to apply classical Riemannian geometric constructions in infinite dimensions. In the last section we describe several open problems concerning the exponential map and its singularities.

\section{The Exponential Map and Conjugate Points on $\mathscr{D}_{\mu}^{s}$}

The differential geometric framework for hydrodynamics outlined above was formulated by Arnold (1966). Its functional analytic foundations were developed subsequently by Ebin and Marsden (1970). For any $s>n / 2+1$ the group $\mathscr{D}_{\mu}^{s}$ has the structure of an infinite-dimensional Hilbert manifold modelled on the Sobolev space of $H^{s}$ divergence-free vector fields on $M$ and the geodesic equation (1.3) of the $L^{2}$ metric is an ordinary differential equation on $\mathscr{D}_{\mu}^{S}$.

This fact provides an important technical advantage of the Lagrangian approach which allows to solve the equations for the particle trajectories (at least on short time intervals) using standard Picard iterations. Consequently, as in finite dimensions, it yields a smooth exponential map, the main object of our study, along with a smooth Levi-Civita connection $\nabla$ and its curvature tensor $\mathcal{R}$. Furthermore, by the inverse function theorem the exponential map is a local diffeomorphism in an open neighbourhood $\mathcal{U}$ of the origin in the tangent space at the identity

$$
\exp _{e}: \mathcal{U} \subset T_{e} \mathscr{D}_{\mu}^{s} \rightarrow \mathscr{D}_{\mu}^{s}, \quad \exp _{e} t v_{0}=\eta(t)
$$

where $\eta(t)$ is the unique $L^{2}$ geodesic with $\eta(0)=e$ and $\dot{\eta}(0)=v_{0}$, see Ebin and Marsden (1970).

Motivated by the problems of hydrodynamic stability Arnold (1966) calculated sectional curvatures of (1.1) when $M$ is the flat 2-torus and showed that they can take on both signs. This led him to conjecture, by analogy with finite dimensions, that positive curvatures are related to the existence of conjugate points in the volumorphism group, see also Arnold (2004), Arnold and Khesin (1998). Because the curvature tensor $\mathcal{R}$ of (1.1) is known to be a continuous multilinear operator in the $H^{s}$ topology, conjugate points in $\mathscr{D}_{\mu}^{s}$ can be studied using the equation of geodesic deviation and the Jacobi fields. ${ }^{2}$ First examples of conjugate points in $\mathscr{D}_{\mu}^{s}$ were constructed in Misiołek (1993), Misiołek (1996) and further examples were given by Shnirelman (1994) and Preston (2006). Epi-conjugate points that are not mono-conjugate and other pathological sit-

\footnotetext{
${ }^{2}$ For these results we refer to Misiołek (1993).
} 
uations were shown to occur in 3D hydrodynamics, cf. Ebin et al. (2006), Preston (2006). On the other hand the 2D case turns out to be entirely different.

Lemma 1 The weak-Riemannian exponential map in 2D hydrodynamics is a nonlinear Fredholm map. More precisely, for any $v \in T_{e} \mathscr{D}_{\mu}^{s}$ the derivative $d \exp _{e}(v)$ is a bounded Fredholm operator of index zero between the spaces $T_{e} \mathscr{D}_{\mu}^{s}$ and $T_{\eta} \mathscr{D}_{\mu}^{s}$ where $\eta=\exp _{e} v$.

Proof See Ebin et al. (2006, Th. 1).

In particular, observe that mono-conjugate points and epi-conjugate points along any $L^{2}$ geodesic in $\mathscr{D}_{\mu}^{s}$ must coincide. Note also that the result of Wolibner (1933) on global existence and uniqueness of solutions to the Euler equations in 2D implies that the exponential map can be defined on the whole tangent space $T_{e} \mathscr{D}_{\mu}^{s}$. In order to prove our Theorem we will need three more lemmas.

Lemma 2 Let $v, w \in T_{e} \mathscr{D}_{\mu}^{s}$ and let $c(t)$ be a smooth curve joining $c(0)=0$ with $c(1)=v$. Then we have

1. $\langle v, w\rangle_{L^{2}}=\left\langle d \exp _{e}(v) v, d \exp _{e}(v) w\right\rangle_{L^{2}}$

2. if $\sigma(t)=\exp _{e} c(t)$ and $\gamma(t)=\exp _{e}$ tv then $L(\gamma) \leq L(\sigma)$.

Proof The first part is an $L^{2}$ version of the classical Gauss Lemma and can be proved as in finite dimensions. Let $Y(t)=d \exp _{e}(t v) t w$ be the Jacobi field along $\gamma(t)=\exp _{e} t v$ with $Y(0)=0$ and $\nabla_{\dot{\gamma}} Y(0)=w$. Since

$$
\frac{d^{2}}{d t^{2}}\langle Y, \dot{\gamma}\rangle_{L^{2}}=\langle R(Y, \dot{\gamma}) \dot{\gamma}, \dot{\gamma}\rangle_{L^{2}}=0
$$

we decompose the Jacobi field into $Y=Y^{\top}+Y^{\perp}$ where $Y^{\top}(t)=t\langle v, w\rangle_{L^{2}} \dot{\gamma}(t)$ and $\left\langle Y^{\perp}(t), \dot{\gamma}(t)\right\rangle_{L^{2}}=0$. Without loss of generality we are assuming that $\|\dot{\gamma}(t)\|_{L^{2}}=1$. We have

$$
\langle v, w\rangle_{L^{2}}=\left\langle\dot{\gamma}(1), Y^{\top}(1)\right\rangle_{L^{2}}=\langle\dot{\gamma}(1), Y(1)\rangle_{L^{2}}=\left\langle d \exp _{e}(v) v, d \exp _{e}(v) w\right\rangle_{L^{2}}
$$

and in particular $\left\|d \exp _{e}(v) v\right\|_{L^{2}}=\|v\|_{L^{2}}=1$.

For the second part introduce polar coordinates $(r, \tilde{c})$ by setting $c(t)=r(t) \tilde{c}(t)$ where $r(t)=\|c(t)\|_{L^{2}}$ and $\|\tilde{c}(t)\|_{L^{2}}=1$. We continue to assume that $v$ has unit $L^{2}$ norm and consider a two-parameter family of curves $\alpha(t, s)=\exp _{e}(s \tilde{c}(t))$ where $s \in$ $(-\delta, \delta)$ for some small $\delta>0$. Observe that $r(0)=0, r(1)=1$ and $\alpha(t, r(t))=\sigma(t)$ so that

$$
\dot{\sigma}(t)=\frac{\partial \alpha}{\partial t}(t, r(t))+\frac{\partial \alpha}{\partial s}(t, r(t)) \dot{r}(t)
$$

Then, we have

$$
\frac{\partial \alpha}{\partial s}(t, s)=d \exp _{e}(s \tilde{c}(t)) \tilde{c}(t) \text { and } \frac{\partial \alpha}{\partial t}(t, s)=d \exp _{e}(s \tilde{c}(t)) \dot{\tilde{c}}(t)
$$


and since $\tilde{c}(t)$ and its time derivative are $L^{2}$-orthogonal applying the Gauss Lemma above gives

$$
\left\langle\frac{\partial \alpha}{\partial t}(t, r(t)), \frac{\partial \alpha}{\partial s}(t, r(t))\right\rangle_{L^{2}}=0
$$

Using this and the fact that $d \exp _{e}$ is an isometry of the $L^{2}$ metric in the radial direction, we now obtain

$$
\|\dot{\sigma}(t)\|_{L^{2}}^{2}=\left\|\frac{\partial \alpha}{\partial t}(t, r(t))\right\|_{L^{2}}^{2}+\left\|\frac{\partial \alpha}{\partial s}(t, r(t)) \dot{r}(t)\right\|_{L^{2}}^{2} \geq|\dot{r}(t)|^{2}
$$

from which integrating in $t$ we get

$$
L(\sigma) \geq \int_{0}^{1}|\dot{r}(t)| d t \geq r(1)-r(0)=\int_{0}^{1}\|\dot{\gamma}(t)\|_{L^{2}} d t=L(\gamma)
$$

Next, we have the following result. ${ }^{3}$

Lemma 3 Any finite geodesic segment in $\mathscr{D}_{\mu}^{s}$ contains at most finitely many conjugate points.

Proof This result is a consequence of the hydrodynamical analogue of the Morse Index theorem (cf. Misiołek and Preston 2010, Th. 8.2) according to which the index of a geodesic segment of finite length in the volumorphism group $\mathscr{D}_{\mu}^{s}$ (of a closed surface) is necessarily finite and equal to the number of conjugate points along the segment each counted with its multiplicity.

The last lemma we will need is the following extension of Schauder's invariance of domain result.

Lemma 4 A locally injective smooth Fredholm map of index zero between Banach manifolds is an open map.

Proof Cf. Tromba (1972, Th. 4).

\section{Proof of the Theorem}

Suppose to the contrary that the $L^{2}$ exponential map is injective in some neighbourhood of its conjugate vector $t_{c} v_{0}$ in $T_{e} \mathscr{D}_{\mu}^{s}$. Since it is a Fredholm map of index zero it then follows from Lemma 4 that $\exp _{e}$ is bijective (in fact a homeomorphism) on some open ball $B(\delta)$ of radius $\delta>0$ centered at $t_{c} v_{0}$ onto the open set $\mathcal{V}=\exp _{e} B(\delta)$. We will

\footnotetext{
${ }^{3}$ Lemma 3 can also be proved using a result of A. Shnirelman on analyticity of the $L^{2}$ exponential map and decomposing $d \exp _{e}$ into a sum of an invertible and a compact operator as in Ebin et al. (2006). See also Problem 2 below.
} 
show that together with Lemma 2 this leads to a contradiction with the fact that $\eta(t)$ can be embedded in a family of curves of strictly shorter $L^{2}$ lengths. As before we assume that $\left\|v_{0}\right\|_{L^{2}}=1$.

To this end observe that since by Lemma 3 conjugate points along $\eta(t)$ are isolated we can choose $t_{1}$ and $t_{2}$ such that

$$
0<t_{1}<t_{c}<t_{2}<T \text { and } t_{2}-t_{1}<\delta
$$

for some $T>0$. Furthermore, we can arrange so that $\eta\left(t_{c}\right)$ is the only conjugate point to $e$ in the segment $\eta\left(\left[t_{1}, t_{2}\right]\right) \subset \mathcal{V}$. Let $Y$ be a Jacobi field along $\eta(t)$ with $Y(0)=Y\left(t_{c}\right)=0$ and extend it to a broken Jacobi field by setting $Y(t)=0$ for $t_{c} \leq t \leq T$. Let $\varphi: \mathbb{R} \rightarrow \mathbb{R}$ be any smooth function such that

$$
\varphi\left(t_{c}\right)>0 \text { and } \varphi(t)=0 \text { for } t \in\left[0, t_{1}\right] \cup\left[t_{2}, T\right] .
$$

Recall that the Levi-Civita connection of the $L^{2}$ metric induces a parallel translation operator along curves in $\mathscr{D}_{\mu}^{s}$ which is an isometry of the tangent spaces with their preHilbert structure (1.1) and commutes with $\nabla$, cf. Misiołek (1993). Let $Z$ denote the parallel translation along $\eta(t)$ of the vector $-\nabla_{\dot{\eta}} Y\left(t_{c}\right) \neq 0$ and for any $\varepsilon>0$ set $X_{\varepsilon}=Y+\varepsilon \varphi Z$. Then, a standard calculation of the $L^{2}$ index form using integration by parts gives

$$
\begin{aligned}
I\left(X_{\varepsilon}, X_{\varepsilon}\right)= & \int_{0}^{T}\left(\left\|\nabla_{\dot{\eta}} X_{\varepsilon}\right\|_{L^{2}}^{2}-\left\langle\mathcal{R}\left(X_{\varepsilon}, \dot{\eta}\right) \dot{\eta}, X_{\varepsilon}\right\rangle_{L^{2}}\right) d t \\
= & \int_{0}^{t_{c}}\left(\left\|\nabla_{\dot{\eta}} Y\right\|_{L^{2}}^{2}-\langle\mathcal{R}(Y, \dot{\eta}) \dot{\eta}, Y\rangle_{L^{2}}\right) d t \\
& +2 \varepsilon \int_{t_{1}}^{t_{c}}\left(\varphi^{\prime}\left\langle\nabla_{\dot{\eta}} Y, Z\right\rangle_{L^{2}}-\varphi\langle\mathcal{R}(Y, \dot{\eta}), \dot{\eta}, Z\rangle_{L^{2}}\right) d t+\mathcal{O}\left(\varepsilon^{2}\right) \\
= & -2 \varepsilon \varphi\left(t_{c}\right)\left\|\nabla_{\dot{\eta}} Y\right\|_{L^{2}}^{2}\left(t_{c}\right)+\mathcal{O}\left(\varepsilon^{2}\right)<0
\end{aligned}
$$

provided that $\varepsilon$ is chosen sufficiently small. Next, define a piecewise smooth variation of $\eta(t)$ by

$$
\alpha(s, t)= \begin{cases}\exp _{e}\left(t v_{0}+s\left(d \exp _{e}\left(t v_{0}\right)\right)^{-1} Y(t)\right), & 0 \leq t \leq t_{1} \\ \exp _{\eta_{t}}\left(s X_{\varepsilon}(t)\right), & t_{1} \leq t \leq T\end{cases}
$$

for any $-\delta<s<\delta$. Note that the variation field of $\alpha(s, t)$ is precisely $X_{\varepsilon}$. Thus, choosing $\varepsilon>0$ as in (3.1) we find that

$$
\left.\frac{\partial}{\partial s}\right|_{s=0} L(\alpha(s))=0 \text { and }\left.\frac{\partial^{2}}{\partial s^{2}}\right|_{s=0} L(\alpha(s))=I\left(X_{\varepsilon}, X_{\varepsilon}\right)<0 .
$$

Observe that the equality in the second formula above holds because $\eta(t)$ has $L^{2}$ unit speed and the Jacobi field $Y$ and its covariant derivative are $L^{2}$ orthogonal to 
the geodesic (note that $Y$ vanishes at two distinct points). Consequently, the variation $\alpha(s, t)$ is length decreasing, that is

$$
L(\alpha(s))<L(\eta)
$$

for sufficiently small $s$.

On the other hand observe that, by construction, $\exp _{e}$ is a bijection from the ball $B(\delta)$ onto $\mathcal{V}$. Therefore, for any such $s$ we can also write

$$
\alpha(s, t)=\exp _{e} c_{s}(t)
$$

where

$$
c_{S}(t)= \begin{cases}t v_{0}+s\left(d \exp _{e}\left(t v_{0}\right)\right)^{-1} Y(t), & 0 \leq t \leq t_{1} \\ \left(\left.\exp _{e}\right|_{B(\delta)}\right)^{-1} \alpha(s, t), & t_{1} \leq t \leq t_{2} \\ t v_{0}, & t_{2} \leq t \leq T\end{cases}
$$

Approximating $c_{s}(t)$ in $T_{e} \mathscr{D}_{\mu}^{s}$ by a smooth curve, if necessary, we conclude from Lemma 2 that

$$
L(\alpha(s)) \geq L(\eta)
$$

which contradicts (3.3) and proves the Theorem.

\section{Discussion and Open Problems}

Since the $L^{2}$ exponential map on $\mathscr{D}_{\mu}^{s}$ is the (Lagrangian) solution map for the Euler equations (1.4)-(1.5) further in-depth studies of its analytic and geometric properties are certainly a matter of considerable interest. Let us first point out a possible alternative strategy which was suggested to us by P. Piccione.

Problem 1 Prove the Theorem using techniques of bifurcation theory of Krasnoselskii, see e.g., Krasnoselskii (1964).

It seems plausible that such an approach could in fact produce a stronger result which we can formulate as

Problem 2 Show that the $L^{2}$ exponential maps fails to be radially injective.

A result of this type in the finite-dimensional case can be found in Piccione et al. (2004) while a more Riemannian-oriented strategy was described by B. Schmidt. Another possible approach when $M$ is real analytic would be to apply Shnirelman's result Shnirelman (2012) in order to make use of the powerful methods of Gohberg et al. (1978) developed for holomorphic Fredholm operator-valued functions.

As mentioned above, Fredholmness of the exponential map suggests strong similarities with Riemannian exponential maps on finite dimensional manifolds. Since by Wolibner's result Wolibner (1933) the volumorphism group $\mathscr{D}_{\mu}^{s}$ equipped with the $L^{2}$ 
metric is geodesically complete it is a natural question whether there is a hydrodynamical analogue of the classical Hopf-Rinow theorem. The most complete results on this to date can be found in Shnirelman (1994) and Misiołek and Preston (2010). A related problem of independent interest is the following

Problem 3 Determine the multiplicity of conjugate points along the $L^{2}$ geodesics in $\mathscr{D}_{\mu}^{s}\left(M^{2}\right)$.

Combined with the fact that the exponential map is Fredholm of index zero the answer to this question could help in determining whether $\exp _{e}$ is a covering map, see Misiołek and Preston (2010). Some recent progress in this direction has been made in Benn (2014) in the case of certain stationary geodesics.

Problem 4 Quantify the failure of the $L^{2}$ exponential map on $\mathscr{D}_{\mu}^{s}\left(M^{3}\right)$ to be a Fredholm map.

The failure of Fredholmness in 3D and the possible consequences that this may entail need to be well understood. Explicit examples where this happens can be found in Ebin et al. (2006) and Preston (2006). In fact, it is known that this phenomenon is borderline in that the exponential map of any right-invariant $H^{r}$ metric on $\mathscr{D}_{\mu}^{s}\left(M^{3}\right)$ with $r>0$ is Fredholm of index zero, cf. Misiołek and Preston (2010). The proofs of Fredholmness in the above papers rely essentially on expressing the derivative of $\exp _{e}$ as a sum of an invertible and a compact operator. In the 3D case it may be possible to measure the deviation from compactness using the tools of spectral theory. A closer examination of explicit examples would be very useful.

Problem 5 Investigate the Fredholmness property when $M$ is a two-dimensional manifold with boundary $\partial M$.

The existing Fredholmness results were obtained for closed manifolds. A different sort of difficulty arises when the underlying manifold $M$ has a boundary. Even the situation in 2D has not yet been fully understood although it is known that in this case the kernel of $d \exp _{e}$ must be finite-dimensional, see Ebin et al. (2006), Misiołek and Preston (2010).

Problem 6 Determine the normal forms for the exponential map in the neighbourhood of a regular conjugate point in $\mathscr{D}_{\mu}^{S}\left(M^{2}\right)$.

The result of this paper above can be viewed as a starting point for an in-depth study of the conjugate locus. This should be possible in certain special cases using the tools of singularity theory and the Fredholm property of the exponential map in 2D. For finite dimensional Riemannian manifolds such a study was carried out by Warner in his thesis Warner (1965) and may serve as a useful guide.

Acknowledgments We thank the referees for helpful comments and gratefully acknowledge support from the Erwin Schrödinger Institute for Mathematical Physics in Vienna, Austria, where part of the research for this paper was done. 


\section{References}

Arnold, V.: Sur la geometrie differentielle des groupes de Lie de dimension infinie et ses applications a l'hydrodynamique des fluides parfaits. Ann. Inst. Fourier (Grenoble) 16, 319-361 (1966)

Arnold, V.: Arnold's Problems. Springer/PHASIS, Berlin/Moscow (2004)

Arnold, V., Khesin, B.: Topological Methods in Hydrodynamics. Springer, New York (1998)

Benn, J.: PhD Dissertation, University of Notre Dame (2014)

Benn, J.: Conjugate points on the symplectomorphism group. Ann. Glob. Anal. Geom. (2015). doi:10.1007/ s10455-015-9461-5

Biliotti, L., Exel, R., Piccione, P., Tausk, D.: On the singularities of the exponential map in infinite dimensional Riemannian manifolds. Math. Ann. 336, 247-267 (2006)

Cheeger, J., Ebin, D.: Comparison Theorems in Riemannian Geometry. North Holland, New York (1975)

Ebin, D., Marsden, J.: Groups of diffeomorphisms and the motion of an incompressible fluid. Ann. Math. 92, 102-163 (1970)

Ebin, D., Misiołek, G., Preston, S.: Singularities of the exponential map on the volume-preserving diffeomorphism group. Geom. Funct. Anal. 16, 850-868 (2006)

Gohberg, I., Kaashoek, M., Lay, D.: Equivalence, linearization and decomposition of holomorphic operator functions. J. Funct. Anal. 28, 102-144 (1978)

Grossman, N.: Hilbert manifolds without epiconjugate points. Proc. Am. Math. Soc. 16, 1365-1371 (1965)

Inci, H.: On the Lagrangian formulation of the incompressible Euler equation. arXiv:1301.5994 [math.AP]

Krasnoselskii, M.: Topological Methods in the Theory of Nonlinear Integral Equations. Pergamon, Oxford (1964)

Misiołek, G.: Stability of ideal fluids and the geometry of the group of diffeomorphisms. Indiana Univ. Math. J. 42, 215-235 (1993)

Misiołek, G.: Conjugate points in $\mathcal{D}_{\mu}\left(\mathbb{T}^{2}\right)$. Proc. Am. Math. Soc. 124, 977-982 (1996)

Misiołek, G., Preston, S.: Fredholm properties of Riemannian exponential maps on diffeomorphism groups. Invent. Math. 179, 191-227 (2010)

Morse, M., Littauer, S.: A characterization of fields in the calculus of variations. Proc. Natl. Acad. Sci. USA 18, 724-730 (1932)

Piccione, P., Portaluri, P., Tausk, V.: Spectral flow, Maslov index and bifurcation of semi-Riemannian geodesics. Ann. Glob. Anal. Geom. 25, 121-149 (2004)

Preston, S.: On the volumorphism group, the first conjugate point is always the hardest. Commun. Math. Phys. 267, 493-513 (2006)

Savage, L.: On the crossings of extremals at focal points. Bull. Am. Math. Soc. 49, 467-469 (1943)

Shnirelman, A.: Generalized fluid flows, their approximation and applications. Geom. Funct. Anal. 4, 586-620 (1994)

Shnirelman, A.: On the analyticity of particle trajectories in the ideal incompressible fluid (2012, preprint). arXiv:1205.5837 [math.AP]

Tromba, A.: Some theorems on Fredholm maps. Proc. Am. Math. Soc. 34, 578-585 (1972)

Warner, F.: The conjugate locus of a Riemannian manifold. Am. J. Math. 87, 575-604 (1965)

Wolibner, W.: Un theoréme sur l'existence du mouvement plan d'un fluide parfait, homogéne, incompressible, pendant un temps infiniment long. Math. Z. 37, 698-726 (1933) 